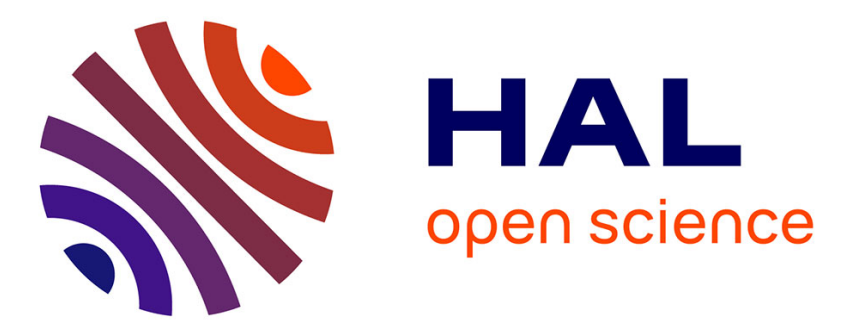

\title{
Affectivité et alexithymie: deux dimensions explicatives des relations entre symptômes dépressifs et anxieux
} Agnes Bonnet, Vincent Bréjard, Aurélie Pasquier, Jean-Louis Pedinielli

\section{To cite this version:}

Agnes Bonnet, Vincent Bréjard, Aurélie Pasquier, Jean-Louis Pedinielli. Affectivité et alexithymie: deux dimensions explicatives des relations entre symptômes dépressifs et anxieux . L'Encéphale, 2012, 38 (3), pp.187-193. 10.1016/j.encep.2011.03.006 . hal-01272703

\section{HAL Id: hal-01272703 \\ https://hal-amu.archives-ouvertes.fr/hal-01272703}

Submitted on 11 Feb 2016

HAL is a multi-disciplinary open access archive for the deposit and dissemination of scientific research documents, whether they are published or not. The documents may come from teaching and research institutions in France or abroad, or from public or private research centers.
L'archive ouverte pluridisciplinaire HAL, est destinée au dépôt et à la diffusion de documents scientifiques de niveau recherche, publiés ou non, émanant des établissements d'enseignement et de recherche français ou étrangers, des laboratoires publics ou privés. 
Affectivité et alexithymie : deux dimensions explicatives des relations entre symptômes dépressifs et anxieux

\section{Affectivity and alexithymia: Two dimensions explicative of the relationshipbetweenanxiety and depressivesymptoms}

A. Bonnet ${ }^{\mathrm{a}}$, V. Bréjard ${ }^{\mathrm{b}}$, A. Pasquier ${ }^{\mathrm{a}}, \mathrm{J}^{-L}$ Pedinielli $^{\mathrm{c}}$

${ }^{a}$ Laboratoire PsyCLE, EA 3273, U.F.R. Psychologie, Université de Provence-France

${ }^{\mathrm{b}}$ LaboratoireLabECD, E.A. 3259, UFR Psychologie, Université de Nantes-France

${ }^{c}$ Laboratoire de Psychopathologie Clinique et Psychanalyse EA 3278, U.F.R. Psychologie, Université de Provence-France

Article publié en juin 2012 dans la revue l'Encéphale (vol. 38, n³, p. 187-193) 


\section{Résumé}

L'objectif de cette étude était de dégager l'existence d'un «noyau commun de vulnérabilité émotionnelle» opérant dans l'anxiété et la dépression; et de préciser les dimensions émotionnelles spécifiques à chacune. Nous avons recruté 317 sujets étudiants. Ils ont rempli un protocole permettant d'évaluer par des échelles d'autoévaluation les symptomatologies dépressive et anxieuse ( SCLR-90), l'affectivité ( EPN-31) et l'alexithymie (TAS-20). Les résultats montrent que l'affectivité négative et la difficulté à identifier les émotions représentent des facteurs commun de vulnérabilité à l'anxiété et à la dépression ; par contre, ces deux symptomatologies se distinguent par la mise en évidence d'éléments spécifiques à chacune. On observe ainsi une faible affectivité positive dans la dépression et une forte activation dans l'anxiété, résultats qui confirment des travaux antérieurs de la littérature. Une particularité réside dans le rôle joué dans la dépression par la pensée orientée vers l'extérieur, dimension opératoire de l'alexithymie.Les limites de l'étude et les ouvertures possibles sont discutées.

Mots-clés : dimensions émotionnelles-alexithymie- vulnérabilité- anxiété-dépression

\section{$\underline{\text { Abstract }}$}

Objectives :The main objective of this study was to support the existence of emotional dimensions common to anxiety and depressive symptomatology, and confirm the common elements of emotional vulnerability, characterized by negative affectivity and alexithymia operation. The second objective of this study was the identification of characteristics specific to each disorder. We made three assumptions:1)there is a significant relationship between anxiety and depressive symptoms, 2) exists on community processes between these two entities, objectified by the sub dimensions of negative affectivity and the difficulty in identifying emotions3) certain dimensions are specific to each disorder.

Method:The study participants were students from 1st to 4th year of the University of Provence. The sample consisted of 317 subjects (77\% female and $23 \%$ G; mean age $=20,61$ \pm 1.55 ), who gave written informed consent and completed questionnaires collectively. We administered to the subjects a protocol consisting of three self-assessment scales to assess emotional dimensions and anxiety and depressive symptomatology. The dimensions of affectivity were assessed by the EPN-31. It consists of 31 items grouped into three factors: positive emotions, negative emotions and feelings of surprise. The emotional functioning was assessed by the scale of the Toronto alexithymia (TAS-20).It allows an overall assessment of the level of alexithymia, as well as three dimensions as represented by specific sub scales: 
difficulty identifying emotions (DIE), the difficulty to differentiate emotions (DDE), and thought oriented 1 outside (PEO). This scale is most used in the assessment of alexithymia. The anxiety and depressive symptoms was assessed by the subscale of anxiety and depression of the SCL90-R. This scale is widely used in screening for psychiatric symptoms, and has been validated internationally.

Statisticalanalysis:we performed descriptive analysis, correlational analysis (correlation of Bravais-Pearson) and hierarchical multiple regression with SPSS 15.

Results validate our assumptions. We observe significant correlations between anxiety and depressive symptomatology and vulnerability factors (negative affectivity, emotional activation and alexithymia). The proposed model can retain common elements and specific dimensions operating respectively for anxiety and depression.

Discussion:Our study reveals the existence of a "common nucleus of vulnerability characterized by negative affectivity associated with difficulty identifying emotions. Specific dimensions appear nevertheless exist, and depression is strongly explained by low positive affect (anhedonia dimension); anxiety associated specificially to emotional activation and finally thought outward, marking the size limitation the imaginary life in alexithymia, appears to operate in depression, perhaps as a mechanism of emotional repression. The involvement of alexithymia in the functioning of the affective disorder is confirmed, this helps to clarify the modalities of therapeutic care that we offer.

$\underline{\text { Key-words }}$ : affective dimensions - alexithymia- emotional vulnerability- anxiety- depression 


\section{Introduction}

Les jeunes adultes constituent actuellement une population particulière étudiée en psychopathologie; la santé mentale de jeunes devient une réalité clinique. Parmi les symptômes les plus fréquemment observés, on retrouve la dépression et les symptômes anxieux, d'ailleurs fréquemment associés.L'anxiété et la dépressionsont des manifestations psychopathologiques qui marquent par leur prévalence dans la population générale; sur une période déterminée, leurs prévalences respectives sont estimées à $2 \%$ pour les troubles anxieux généralisés, entre $6 \%$ et $8 \%$ pour la dépression (19); plus avant, la comorbidité entre anxiété et dépression selon le trouble primaire a pu être observée entre 26\% et 38\%(5).Ces deux types de symptômes représentent des facteurs de détresse émotionnelle, pouvant être mis en relation avec d'autres troubles, telles que les conduites addictives (25). Une étude de 2003 rapporte que l'anxiété est un état pathologique très répandu dans la société actuelle; il représente, en outre, un facteur de risque de la dépression et des conduites addictives.Il faut noter que la présence de telles symptomatologies ne donne pas nécessairement lieu à une démarche thérapeutique (psychologique, psychiatrique) de la part des individus ou encore, à une prise en charge efficace (28).

L'anxiété se définit comme un état émotionnel marqué par la perception imminente d'un danger, une attitude d'attente devant celui-ci et un sentiment de désorganisation lié à la conscience d'une impuissance totale en face de ce danger (29).On qualifie l'anxiété comme la facette psychique de l'angoisse ; elle aussi considérée comme le principal symptômeassocié de la dépression. La dépression peut être considérée comme un symptôme psychopathologique consécutif à desfacteurs de vulnérabilité (tempérament, dysfonctionnement cognitif, personnalité pathologique, dysfonctionnements biologiques). Elle peut également être considérée comme un processus ; en tant que telle, elle définirait un mode de fonctionnement déterminé. Ainsi, des symptômes «non dépressifs » pourraient être considérés comme conséquences d'une lutte contre la dépression sous-jacente. La dépression constituerait un facteur prédictif de l'engagement dans des conduites à risques, et notamment dans l'abus de substances ou la sexualité à risques (2); toutefois, ces travaux (chez l'adolescent) ne permettent pas de préciser si le trouble dépressif était antérieur aux conduites à risques, comorbide ou secondaire à celui-ci.

\section{Modèles psychopathologiques explicatifs de l'anxiété et de la dépression}

Afin de mieux comprendre les relations entre pathologies anxieuses et dépressives, différentes propositions ont été formulées, privilégiant le fonctionnement biochimique, la personnalité, 
ou encore la recherche de dysfonctionnements émotionnels. D'un point de vue psychopathologique, deux tendances semblent se dégager: l'une visant à explorer l'hypothèse d'une vulnérabilité en termes de dimensions spécifiques de la personnalité, l'autre privilégiant une analyse processuelle des troubles de la régulation des émotions.La première approche considère qu'il existe deux grandes dimensions de personnalité touchant le fonctionnement émotionnel: l'affectivité négative et l'affectivité positive renvoient à l'existence d'une disposition émotionnelle (caractéristique stable de la personnalité) sous-tendant le fonctionnement émotionnel d'un sujet. Cette notion rend compte de la propension d'un individu à expérimenter de manière privilégiée des affects positifs ou négatifs $(14 ; 34)$. Elle repose sur un modèle tripartite des émotions: dimensions négative, positive, et activation permettant de rendre compte de la vulnérabilité à présenter un certain nombre de troubles psychopathologiques (troubles dépressifs et anxieux) $(10 ; 34)$. L'affectivité, reprise sous le terme d'émotionnalité(18) est décrite comme un continuum variant d'un pôle négatif à un pôle positif. Cette conception dimensionnelle est d'ailleurs soutenue par différents questionnaires (PANAS, questionnaire de Watson et Clark, EPN-31 (28) qui ont permis de valider l'existence de corrélations importantes entre la dimension émotionnelle négative et des symptômes thymiques telles que l'anxiété et la dépression.La seconde approche s'intéresse aux pathologies du fonctionnement émotionnel. Dans ce champ, l'alexithymie (difficulté à identifier et exprimer les émotions) a fait l'objet de l'essentiel des travaux. Elle correspond à un mode particulier de traitement des émotions, caractérisé par un déficit de représentations mentales de l'éprouvé subjectif (31), se retrouvant chez différentes populations cliniques ( i.e. états de stress post-traumatiques, troubles psychosomatiques, addictions - (27). Elle correspondrait à une dimension secondaire de la personnalité $(23 ; 35)$ expliquant les relations entre l'instabilité émotionnelle tempéramentale ( névrosisme) et certains troubles psychopathologiques.Dans sa dimension émotionnelle, l'alexithymie apparaît comme thymodépendante, contrairement à la dimension cognitive (15). Des travaux actuels montrent l'existence de relations positives entre la difficulté dans l'identification et la description des émotions et les symptomatologies anxieuses et dépressives $(8 ; 17)$ l'intensité émotionnelle (26), ainsi que, dans une perspective adaptative, les conduites à risques et de consommation (4). Elle est retrouvée très fréquemment associée à la dépression chez des sujets présentant une dépendance à l'alcool (16) et plus généralement une consommation excessive de substances psychoactives (20). 


\section{Relations anxiété, dépression et alexithymie}

Les relations entre dépression et alexithymie ont fait l'objet d'une controverse nourrie, entre tenants d'une alexithymie conçue comme un épiphénomène de la dépression, et tenants de l'alexithymie comme fonctionnement distinct de la problématique dépressive. Le débat est actuellement en passe d'être résolu, en raison notamment de travaux portant sur la stabilité relative de l'alexithymie(22). Ainsi, le lien avéré existant entre alexithymie et dépression se trouve précisé par l'évolution temporelle respective des deux pathologies. Dans une étude portant sur une population infraclinique, Berthoz et al.(3) proposent un modèle où l'alexithymie est directement influencée par l'anxiété, elle-même influencée par la dépression. Néanmoins, les relations de causalité suggérées par le modèle ne peuvent être confirmées, du fait de l'utilisation d'analyses statistiques où la réversibilité est possible.

Les principales études réalisées sur les relations entre symptomatologies anxieuses et dépressives ont montré un lien significatif entre ces deux entités, sans toutefois s'intéresser aux relations entre dimensions émotionnelles et pathologies de l'émotion.Peu de travaux abordent les liens spécifiques entre affectivité négative, alexithymie et anxiété/dépression. Existe-t-il une « géométrie » spécifique à chaque symptomatologie ?

Notre objectif principal dans cette étude est de confirmer l'existence d'un «noyau de vulnérabilité émotionnelle » commun aux deux entités $(6 ; 33)$, constitué d'une propension à expérimenter des émotions négatives associé à un fonctionnement alexithymique. Un second objectif est la recherche de caractéristiques spécifiques à chaque trouble dans une perspective dimensionnelle. Nous formulons donc trois hypothèses :1) il existe une relation significative entre symptômes anxieux et dépressifs, 2) il existe une relative communauté de processus entre ces deux entités, objectivées par les sous dimensions de l'affectivité négative et de la difficulté à identifier les émotions 3) certaines sous dimensions sont spécifiques à chaque trouble.

\section{Matériel et méthode}

\section{Sujets}

Les participants à l'étude sont des étudiants de lère à 4ème année de l'Université de Provence. L'échantillon est constitué de 317 sujets $(77 \% \mathrm{~F}$ et $23 \% \mathrm{G}$; âge moyen $=20$, $61 \pm 1,55)$, qui ont donné par écrit leur consentement éclairé et rempli les questionnaires collectivement. 


\section{Evaluation}

Les dimensions de l'affectivité ont été évaluées par 1'EPN-31. Cette échelle permet de mesurer les trois dimensions des émotions en référence au modèle tripartite de Watson et Clark. Elle est constituée de 31 items regroupés en trois facteurs : émotions positives, émotions négatives et émotions de surprise. La consistance interne varie entre 0,80 et 0,95 pour les trois facteurs. Une corrélation négative a été retrouvée entre émotions positives et sévérité des symptômes dépressifs, et une corrélation positive a été observée entre émotions négatives et sévérité des symptômes anxieux et dépressifs. Le fonctionnement émotionnel a été évalué par l'échelle d'alexithymie de Toronto (TAS-20). C'est une échelle d'autoévaluation comportant 20 affirmations sur une échelle en cinq points (de « désaccord complet » à «accord complet »). Chaque item est côté de 1 à 5 et le score global est de 100, le score à partir duquel un individu est considéré comme alexithymique est de 56. Elle permet une évaluation globale du niveau d'alexithymie, ainsi que de trois sous dimensions représentées par des sous échelles spécifiques : la difficulté à identifier ses émotions (DIE), la difficulté à différencier les émotions (DDE), et la pensée orientée vers l'extérieur (PEO) Cette échelle est la plus utilisée dans l'évaluation de l'alexithymie. Ses qualités métrologiques sont fiables et ont été analysées dans de nombreux travaux.

La symptomatologie anxieuse et dépressive a été évaluée par les sous échelles d'anxiété et de dépression de la SCL90-R. Cette échelle est largement utilisée dans le dépistage de symptômes psychiatriques, et a fait l'objet d'une validation internationale. Ses qualités psychométriques sont satisfaisantes(12).

\section{Démarche d'analyse des données}

Différents traitements statistiques ont été réalisés. En premier lieu des analyses descriptives portant sur l'existence de différences liées au sexe. Ensuite des analyses corrélationnelles (corrélations de Bravais-Pearson) ont été réalisées afin de tester les relations entre les différentes variables; enfin des régressions multiples hiérarchiques ont été calculées, afin de déterminer la pertinence de différents modèles de relations entre variables explicatives (critère) et symptômes. Les traitements statistiques ont été réalisés avec le logiciel SPSS 15.

\section{Résultats}

Le tableau 1 présente les analyses descriptives et les différences inter sexes. On observe ainsi que les femmes sont plus anxieuses et plus déprimées que les hommes, et présentent un niveau d'alexithymie supérieur. Plus précisément, seule la dimension « difficulté à identifier les émotions » et le score général d'alexithymie sont concernés par cette différence. En 
revanche, on n'observe pas de différence significative sur les dimensions émotionnelles de l'affectivité négative, positive et de l'activation.

\section{$\underline{\text { Tableau } 1}$}

L'analyse des relations entre les différentes variables montre l'existence de plusieurs liaisons significatives. Celles-ci sont présentées dans le tableau 2. On retrouve une forte corrélation ( $\mathrm{r}$ >.50) positive entre anxiété et dépression, ce qui signifie que la présence chez un sujet, de l'un ou l'autre trouble, a une forte probabilité d'être associéeau second. Ces deux symptômes entretiennent,en outre,des relations fortes $(r>.50)$, modérées $(r>.30)$ à faibles $(r>.10)$ avec certains facteurs de vulnérabilité potentiels.Ainsi, l'affectivité négative présente une forte relation avec l'anxiété et la dépression, tandis que l'affectivité positive n'est liée négativement et modérément- qu'à la dépression. Enfin, l'activation émotionnelle entretient une relation modérée avec l'anxiété, et faible avec la dépression. Il existe également des relations significatives entre symptômes affectifs et alexithymie, notamment dans sa composante émotionnelle : difficulté à identifier les émotions et difficulté à décrire les émotions.

\section{$\underline{\text { Tableau } 2}$}

Afin d'analyser les contributions relatives des différentes dimensions de l'affectivité et de l'alexithymie au niveau des symptomatologies dépressives et anxieuses, nous avons testé le même modèle global incluant l'affectivité négative, positive et l'activation, et les sous dimensions de l'alexithymie, pour chaque modalité d'expression symptomatique. Les résultats sont présentés dans le tableau 3. Concernant l'anxiété, seules contribuent de manière significative les dimensions suivantes en expliquant $43 \%$ de la variance : le sexe, l'affectivité négative, l'activation émotionnelle et les deux sous dimensions de l'alexithymie « difficulté à identifier les émotions » et «pensées orientées vers l'extérieur ». La dépression partage avec l'anxiété les dimensions suivantes: sexe, affectivité négative, difficulté à identifier les émotions, et inclut une dimension spécifique, l'affectivité positive, pour constituer un modèle expliquant $51 \%$ de la variance. En revanche, l'absence de contribution de l'activation émotionnelle et de la «pensée orientée vers l'extérieur»doit être soulignée. Ainsi peut-on observer que les deux dimensions spécifiques de l'affectivité négative et de la difficulté à 
identifier les émotions constituent un point de «recouvrement» des problématiques dépressives et anxieuses.

\section{$\underline{\text { Tableau } 3}$}

\section{Discussion}

Notre ambition dans cette étude était,premièrement, de répliquer les résultats obtenus dans les études antérieures analysant les relations entre troubles anxieux et dépressifs, et secondairement de préciser ces relations par l'analyse de la contribution possible, à ces relations, d'une pathologie du fonctionnement émotionnel. Nous avions formulé deux objectifs consistant, d'une part, à mettre en évidence l'existence d'une vulnérabilité psychopathologique aux symptomatologies anxieuses et dépressives; d'autre part, à dégager leurs caractéristiques spécifiques dans une perspective dimensionnelle. Les résultats confirment les hypothèses formulées. L'anxiété et la dépression présentent un «noyau psychopathologique commun » constitué d'une forte affectivité négative et d'une difficulté significative à identifier les émotions. La valence et l'activation de l'expérience émotionnelle sont ainsi en lien avec ces deux symptomatologies. La présence d'éléments non spécifiques tant du point de vue symptomatique que psychopathologique est confirmée $(9 ; 30)$. On observe, de plus,un effet possible de l'alexithymie dans cette relation entre anxiété et dépression. Le rôle de la difficulté à identifier les émotions peut-être ici interrogé dans sa contribution à un état de malaise interne indifférencié, etpourrait être,en réalité, l'élément central opérant dans les relations entre ces deux types de symptomatologies.Plus avant, les spécificités propres à chaque expression symptomatique apportent des éléments complémentaires. Conformément aux données de la littérature, nos résultats soulignent la présence d'une dimension anhédonique (représentée par une faible affectivité positive) associée à la difficulté à identifier les émotions dans la dépression. L'anhédonie que l'on peut rattacher à l'émoussement affectif a été en effet, déjà associée à l'alexithymie dans ce sens7;11;32). Par ailleurs, l'anxiété est associée à une forte activation émotionelle( hyperarousal), là encore, conformément à la littérature. L'intensité émotionnelle dans l'anxiété est associée à la dimension d'éveil physiologique inhérente à cette émotion (1). En revanche, on observe, pour la dépression, une contribution négative de la pensée orientée vers l'extérieur, au modèle, ce qui va à l'encontre de résultats obtenus dans une étude précédente(24). Ceci pourrait s'expliquer par le caractère infra clinique des symptômes de notre échantillon, mais pourrait égalementtémoigner d'une opération défensive visant à éviter 
l'activation psychique en excès. Cette dimension de l'alexithymie rend compte d'un fonctionnement émotionnel marqué par un mécanisme de répression des affects (7),pouvant donner lieu à l'expression d'une «pensée( dite) opératoire» (21).Ces résultats nous permettent ainsi de préciser et d'étayer les travaux permettant de soutenir les spécificités de l'anxiété et de la dépression, par la reconnaissance des dimensions du fonctionnement émotionnel et de leur articulation.

Plusieurs limites méthodologiques invitent cependant à rester mesurés dans la généralisation de nos résultats. En effet, l'étude par sa construction transversale, ne permet pas de soutenir avec certitude le sens de la relation entre dimensions de personnalité, alexithymie, et symptômes dépressifs ou anxieux.Ainsi, une interprétation alternative pourrait être que ce sont les symptômes anxieux et dépressifs qui amènent les sujets à recourir à un fonctionnement alexithymique, les conduisant alors à privilégier l'éprouvé stable d'affects négatifs ou positifs. Cette alternative trouve néanmoins peu d'étayages tant sur les plans théoriques qu'empiriques, et renvoie au débat sur les relations entre alexithymie et anxiétédépression, auquel des éléments de réponse ont déjà été apportés (13;22 ;24). Une autre limite est constituée par notre échantillon comportant un déséquilibre garçon/fille d'une part, et par l'absence de groupe «clinique»d'autre part, limitant la portée de nos conclusions. Néanmoins, une étude antérieure comportant un échantillon composé de patients déprimés et anxieux retrouve des résultats similaires concernant les relations entre dimensions de l'affectivité et symptômes anxieux et dépressifs (6). Enfin, des travaux à venir devront compléter l'évaluation par auto questionnaire en y associant des hétéro évaluations cliniques reposant, par exemple,à partit d'entretiens semi structurés.

\section{Conclusion}

Cette recherche, la première à notre connaissance portant sur l'étude des dimensions de l'affectivité en lien avec l'alexithymie dans l'analyse des relations entre symptômes anxieux et dépressifs, permet de souligner la nette implication d'un fonctionnement alexithymique dans les «troubles de l'affectivité ». La difficulté à identifier les émotions apparaît constituer une dimension opératoire du fonctionnement émotionnel. Ce constat doit à notre sens être mis en perspective avec la faible demande psychothérapeutique chez les sujets présentant ces problématiques, sujets demandant toutefois une aide pharmacologique, d'ailleurs souvent nécessaire. Néanmoins, ne peut-on faire l'hypothèse qu'un travail d'élaboration pourrait conduire ces patients à s'autoriser une interrogation sur leurs éprouvés subjectifs ? L'impact potentiel dans une perspective préventive d'une authentique décompensation est posé. 


\section{Références}

1. Amado-Boccara I, Donnet, D., Olié, J-P. . La notion d'humeur en psychologie. L'Encéphale, 1993; XIX, : 117-22.

2. Bancroft J, Janssen, E., Carnes, L., Goodrich, D., Strong, D., Long, J.S. Sexual activity and risk taking in young heterosexual men: the relevance of sexual arousability, mood, and sensation seeking. Journal of sexuality research 2004; 41 (2):181-92.

3. Berthoz S, Consoli S, Perez-Diaz F et al. Alexithymia and anxiety: compounded relationships? A psychometricstudy. EuropeanPsychiatry 1999; 14 (7):372-8.

4. Brejard V, Pedinielli JL, Rouan G. Hypothèse d'un dysfonctionnement émotionnel chez des adolescents présentant des comportements à risqué : une étude exploratoire. L'Encéphale 2006; 32 (4, Part 1):413-20.

5. Brochier G. Coexistence des troubles anxieux et dépressifs Paris: Agence Nationale pour l'Evénement Médical.

6. Burns DD, Eidelson RJ. Why are depression and anxiety correlated? A test of the tripartite model. Journal of Consulting and Clinical Psychology 1998; 66 (3):461-73.

7. Carton S. La répression émotionnelle et son rôle en psychopathologie Psychologie Française 2006; 51 (2): 123-39.

8. Ciarrochi J, Scott, G., Deane, F.P., Heaven, P.C.L. Relations between social and emotional competence and mental health : a construct validation study. Personality and Individual Differences 2003; 35 (8):1947-63.

9. Clark DA, Steer RA, Beck AT. Common and specific dimensions of self-reported anxiety and depression: Implications for the cognitive and tripartite models. Journal of Abnormal Psychology 1994; 103 (4):645-54.

10. De Gucht V, Fischler, B., Heiser, W. Neuroticism, alexithymia, negative affect, and positive affect as determinants of medically unexplained symptoms. Personality and IndividualDifferences 2004; 36:1655-67.

11. Deborde AS, Berthoz, S., Perdereau, F., Godart, N., Corcos, M., Jeammet, P. Validité du questionnaire d'alexithymie de Bermond et Vorst : étude chez des sujets présentant des troubles du comportement alimentaire et chez des témoins. L'Encéphale 2004; 30:464-73.

12. Derogatis LR. Symptom Checklist-90-R (SCL-90-R) : Administration, scoring and procedures manual, . 1994.

13. EspinaEizaguirre A, Ortego Saenz de Cabezon A, Ochoa de Alda I et al. Alexithymia and its relationships with anxiety and depression in eating disorders. Personality and Individual Differences 2004; 36 (2):321-31.

14. Eysenck HJ. The biological dimensions of personality. In: LA Pervin, editor, translator and editor Handbook of personality: theory and research. New York: Guilford; 1990; p. 224-76.

15. Fargès F, Fargès, S. Alexithymie et substances psychoactives : revue critique de la littérature. Psychotropes 2002; 8 (2):47-74.

16. Haviland MG, Mac Murray, J.P., Cummings, M.A. The relationship between alexithymia and depressive symptoms in a sample of newly abstinent alcoholic inpatients. . Psychotherapy psychosomatics 1988; 49:37-40.

17. Honkalampi K, Hintikka, J., Tanskanen, A., Lehtonen, J., Viinamäki, H. Depression is strongly associated with alexithymia in the general population. Journal of Psychosomatic Research 2000; 48:99-104.

18. Larsen RJ, Diener, E. A multitraitmultimethod examination of affect structure: hedonic level and emotional intensity. Personality and Individual Differences 1985; 6 (5):631-6. 
19. Lépine JP, Gasquet, I., Kovess, V., Arbabzadeh-Bouchez, S., Nègre-Pagès, L., Nachbaur, G., Gaudin, A.F. Prevalence and comorbidity of psychiatric disorders in the French general population. Encephale 2005; 31 (2):192-94.

20. Loas G, Otmani O, Lecercle $\mathrm{C}$ et al. Relationships between the emotional and cognitive components of alexithymia and dependency in alcoholics. PsychiatryResearch 2000; 96 (1):63-74.

21. Luminet O. Psychologie des émotions. Confrontation et évitement. Bruxelles: DeBoeck; 2002.

22. Luminet O, Bagby RM, Taylor GJ. An evaluation of the absolute and relative stability of alexithymia in patients with major depression. Psychotherapy \& Psychosomatics 2001; 70 (5):254-60.

23. Luminet O, Bagby, R.M., Wagner, H., Taylor, G.J., Parker, J.D.A. Relation between alexithymia and the five-factor model of personality: a facet-level analysis. Journal of Personality Assessment 1999; 73:345-58.

24. Marchesi C, Brusamonti E, Maggini C. Are alexithymia, depression, and anxiety distinct constructs in affective disorders? Journal of Psychosomatic Research 2000; 49 (1):43-9.

25. Olié JP, Gourion, D., Canceil, O., Lô, H. Physiological adolescence, pathological adolescence. Bull AcadNatl Med. 2006; 190 (8):1643-51.

26. Pasquier A, Bonnet, A., Pedinielli, J.L. Fonctionnement cognitivo-émotionnel : le rôle de l'intensité émotionnelle chez les individus anxieux. Annales MedicoPsychologiques 2009; 167 (9):649-56.

27. Pedinielli J-L. Psychosomatique et Alexithymie. Paris: Presses Universitaires de France; 1992.

28. Pélissolo A, Rolland JP, Perez-Diaz F et al. Évaluation dimensionnelle des émotions en psychiatrie : validation du questionnaire Émotionnalité positive et négative à 31 items (EPN-31). L'Encéphale 2007; 33 (3):256-63.

29. Pichot P. Les dépressions : problèmes de vocabulaire et nosologie. . Paris: Masson; 1978.

30. Ruth S, Mehrotra S. Differentiating depression and anxiety: Psychometric implications of the tripartite model of affect. Journal of Personality and Clinical Studies 2001; 17 (1):9-18.

31. Sifneos PE. The prevalence of "alexithymic" characteristics in psychosomatic patients. Psychoth Psychosomatics 1973; Vol. 22 (2):255-62.

32. Speranza M, Corcos, M., Stephan, P., Loas, G., Pérez-Diaz, F., Lang, F., Venisse, J.L., Bizouard, P., Flament, M., Halfon, O., Jeammet, P. Alexithymia, Depressive Experiences, and Dependency in Addictive Disorders. Substance Use and Misuse 2004; 39 (4):551-79.

33. Watson D, Clark LA, Weber $\mathrm{K}$ et al. Testing a tripartite model: II. Exploring the symptom structure of anxiety and depression in student, adult, and patient samples. Journal of Abnormal Psychology 1995; 104 (1):15-25.

34. Watson D, Clark, L.A. Negative affectivity: the disposition to experience aversive emotional states. Psychological Bulletin 1984; 96:465-90.

35. Zimmermann G, Salamin V, Reicherts M. L'alexithymie aujourd'hui: Essai d'articulation avec les conceptions contemporaines des émotions et de la personnalité. PsychologieFrançaise 2008; 53 (1):115-28. 
Tableau 1: Comparaison des sujets masculins et féminins pour l'âge, les symptômes anxieux et dépressifs, l'affectivité et l'alexithymie

\begin{tabular}{|c|c|c|c|c|c|c|c|c|}
\hline & $\mathrm{M}$ & & $\mathrm{F}$ & & Total & & & \\
\hline & $\mathrm{M}$ & (S.D.) & $\mathrm{M}$ & (S.D.) & $\mathrm{M}$ & (S.D.) & $t(2,313)$ & $p$ \\
\hline Age & 20,88 & $(1,61)$ & 20,53 & $(1,53)$ & 20,61 & $(1,55)$ & 1,68 & 0,09 \\
\hline SCL Dépression & 7,75 & $(6,53)$ & 10,61 & $(8,73)$ & 9,95 & $(8,34)$ & $-2,56$ & 0,01 \\
\hline SCL Anxiété & 4,77 & $(4,97)$ & 6,68 & $(6,20)$ & 6,26 & $(5,98)$ & $-2,38$ & 0,02 \\
\hline EPN PA & 48,63 & $(11,74)$ & 48,37 & $(9,38)$ & 48,48 & $(9,99)$ & 0,19 & 0,85 \\
\hline EPN NA & 55,23 & $(20,28)$ & 56,22 & $(18,04)$ & 56,02 & $(18,54)$ & $-0,40$ & 0,69 \\
\hline EPN AE & 9,01 & $(3,45)$ & 8,3 & $(3,71)$ & 8,46 & $(3,65)$ & 1,43 & 0,15 \\
\hline TAS DIE & 15,65 & $(6,00)$ & 17,92 & $(5,95)$ & 17,38 & $(6,01)$ & $-2,83$ & 0,01 \\
\hline TAS DDE & 13,42 & $(5,07)$ & 14,7 & $(5,24)$ & 14,40 & $(5,23)$ & $-1,82$ & 0,07 \\
\hline TAS POE & 16,76 & $(4,14)$ & 16,66 & $(5,01)$ & 16,69 & $(4,81)$ & 0,16 & 0,88 \\
\hline TAS Total & 45,83 & $(11,29)$ & 49,28 & $(12,46)$ & 48,47 & $(12,25)$ & $-2,09$ & 0,04 \\
\hline
\end{tabular}

SCL: Symptom Check-List; EPN: Echelle d'émotions Positives et Négatives;

TAS: Toronto AlexithymiaScale 
Tableau 2 : Corrélations entre âge, symptômes dépressifs, anxieux, et variables explicatives : dimensions émotionnelleset alexithymie.

\begin{tabular}{|c|c|c|c|c|c|c|c|c|c|c|c|}
\hline & & 1 & 2 & 3 & 4 & 5 & 6 & 7 & 8 & 9 & 10 \\
\hline 1 & Age & 1,00 &,$- 11(*)$ &,$- 17(* *)$ & $-0,01$ &,$- 16(* *)$ & $-0,09$ & $-0,10$ & $-0,09$ & 0,10 & $-0,05$ \\
\hline 2 & SCL Dépression & & 1,00 &, $59(* *)$ &,$- 33(* *)$ &, $63(* *)$ &, $23(* *)$ &, $48(* *)$ &, $31(* *)$ & $-0,01$ &, $36(* *)$ \\
\hline 3 & SCL Anxiété & & & 1,00 & $-0,07$ &, $58(* *)$ &, $42(* *)$ &, $46(* *)$ &, $25(* *)$ & $-0,09$ &, $23(* *)$ \\
\hline 4 & EPN PA & & & & 1,00 & $-0,02$ &, $18(* *)$ &,$- 16(* *)$ &,$- 20(* *)$ &,$- 11(*)$ &,$- 21(* *)$ \\
\hline 5 & EPN NA & & & & & 1,00 &, $38(* *)$ &, $49(* *)$ &, $31(* *)$ & $-0,09$ &, $34(* *)$ \\
\hline 6 & EPN AE & & & & & & 1,00 &, $27(* *)$ &, $21(* *)$ & 0,02 &, $23(* *)$ \\
\hline 7 & TAS DIE & & & & & & & 1,00 &, $59(* *)$ &, $22(* *)$ &, $83(* *)$ \\
\hline 8 & TAS DDE & & & & & & & & 1,00 &, $26(* *)$ &, $82(* *)$ \\
\hline 9 & TAS POE & & & & & & & & & 1,00 &, $61(* *)$ \\
\hline 10 & TAS Total & & & & & & & & & & 1,00 \\
\hline
\end{tabular}

$* p<0.05 ; * * p<0.01$

SCL: Symptom Check-List; EPN: Echelle démotions Positives et Négatives; TAS: Toronto

AlexithymiaScale 
Tableau 3 : Résultats des analyses de régression multiples: dimensions émotionnelles et alexithymie comme facteurs contribuant aux symptomatologies anxieuses et dépressives.

\begin{tabular}{|c|c|c|c|c|c|c|}
\hline & \multicolumn{3}{|c|}{$\begin{array}{l}\text { Symptômes } \\
\text { anxieux }^{\text {a }}\end{array}$} & \multicolumn{3}{|c|}{ Symptômes dépressifs ${ }^{b}$} \\
\hline & $\bar{\beta}$ & $t$ & $p$ & $\beta$ & $t$ & $p$ \\
\hline Age & $-0,06$ & $-1,27$ & 0,20 & 0,00 & $-0,03$ & 0,97 \\
\hline Sexe & 0,10 & 2,32 & 0,02 & 0,11 & 2,75 & 0,006 \\
\hline EPN PA & $-0,09$ & $-1,97$ & 0,09 & $-0,32$ & $-7,51$ & $<0,0001$ \\
\hline EPN NA & 0,36 & 6,60 & $<0,0001$ & 0,52 & 10,35 & $<0,0001$ \\
\hline EPN AE & 0,25 & 5,18 & $<0,0001$ & 0,069 & 1,52 & 0,13 \\
\hline TAS DIE & 0,24 & 3,98 & $<0,0001$ & 0,16 & 2,9 & 0,004 \\
\hline TAS DDE & $-0,07$ & $-1,29$ & 0,20 & $-0,03$ & $-0,66$ & 0,51 \\
\hline TAS POE & $-0,09$ & $-1,94$ & 0,05 & $-0,02$ & $-0,57$ & 0,80 \\
\hline
\end{tabular}

${ }^{a} R^{2}$ ajusté $=0,43 p<0,0001$

${ }^{b} R^{2}$ ajusté $=0,51 p<0,0001$ 
